\title{
Design and Developmental Research on the VV\&A of Complex Simulation System
}

\author{
Li Liu', Yan Bin Li, Da Wu, Lin Zhang, Ning Wang \\ Air Force Engineering University, Xi'an China
}

\begin{abstract}
The Verification, Validation and Accreditation(VV\&A) of a complex simulation system is a complex systems engineering. Based on the brief introduction to the concept of VV\&A, this paper puts forward its design principles, approaches and basic contents, expounds the typical developing process and predicts its up-to-date technology developing trend of complex simulation system.
\end{abstract}

Keywords- Complex Systems; Modeling and Simulation(M\&S); Verification, Validation and Accreditation(VV\&A)

\section{VV\&A CONCEPTION}

Complex simulation system reliability can be judged by verification, certificated by validation, so that the complex simulation system can serve a specific purpose, this process is called verification, validation and accreditation, which is referred to as VV\&A.

Verification is to determine whether or not the simulation system model and its simulation realization accurately have expressed the iterative process which the developer needs. It focuses on the inspection of the simulation modeling process, namely whether the code and logic are correct and whether the expected functions have been realized.

Validation is to confirm the authenticity of simulation model, a thorough inspection of mathematic and logic models. It uses the data from real world and creditable source comparing with the output and behaviors of simulation system to confirm whether it is vivid or not. Therefore, the fundamental methods of validation are experiment, observation and comparison, the most obvious purpose is to provide a set of credibility evidence of models or simulation for special applications.

Accreditation is to address the issue of whether simulation system is suitable for a particular application and whether to accept it or not by the government or authority. The process is based on the results of VV\&A, and compared with an acceptable standard.

Verification involves the question of whether or not develop the simulation system correctly, validation involves the question of whether or not develop a correct system model, accreditation involves the issue of whether can use the simulation system. The relationship between them is shown in Figure1.

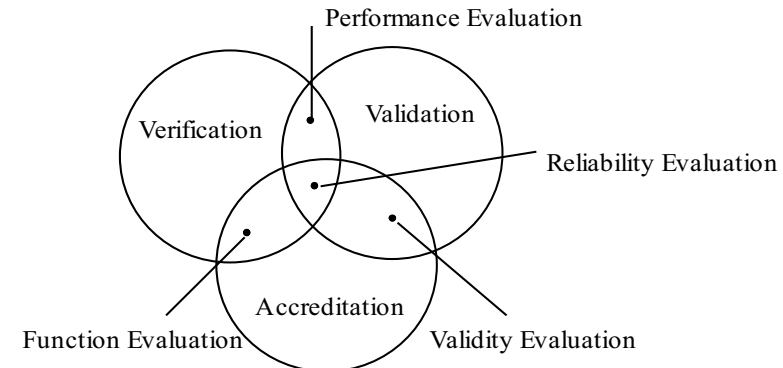

Figure1 the relationship between the VV\&A

\section{THE PRINCIPLES, STEPS AND BASIC CONTENTS OF VV\&A SCHEME DESIGN}

The result of complex simulation system VV\&A highly depends on the established schemes. The establishment process of scheme must have the principles as follows:

(1) The VV\&A scheme should cover the total lifecycle of simulation system;

(2) Considering comprehensively the influential factor of VV\&A comprehensively;

(3) The VV\&A scheme must be feasible;

(4) Some VV\&A work which has large influence on the creditability should be put in the first position to enhance the effectiveness under limited input;

\footnotetext{
${ }^{a}$ Corresponding author: 11200010@126.com
} 
(5) Comprehending sufficiently the importance of VV\&A work, then establishing the most effective VV\&A scheme according to the reality;

(6) Modifying the scheme according to the specific requirement based on the standard VV\&A process model.

The VV\&A scheme design steps as follows:

Collecting information $\rightarrow$ Analyzing the risk $\rightarrow$ Confirming the task of VV\&A $\rightarrow$ Modifying VV\&A activities $\rightarrow$ Designing VV\&A scheme in detail $\rightarrow$ Reviewing VV\&A scheme $\rightarrow$ Amending VV\&A scheme $\rightarrow$ Submitting the document of VV\&A plan

The basic contents of VV\&A plan include two parts: $\mathrm{V} \& \mathrm{~V}$ and validation.

To V\&V: 1)The purpose and description of application (2) The background of simulation system (3)The definition and references (4)The correlative people and their duty of V\&V(5)The expected use of simulation system(6)The information source of VV\&A implement (7)The description of V\&V complete activities projected (8)The tool(9)Budget(10)The V\&V report; (11)The appendix;

To the validation:(1)The purpose(2)The background of simulation system(3)The definition and references(4)The correlative people and their duty of V\&V(5)The schedule, milestone and resource(6) The expected use of simulation system(7)The information source of VV\&A implement(8)The acceptable standard (9)The validation methods.

\section{THE TYPICAL DEVELOPING PROCESS OF VV\&A}

\section{A. The lifecycle $V V \& A$ developing process based on DIS}

The lifecycle developing process of DIS is as follows:

Planning drills and defining requirement $\rightarrow$ Designing, developing and testing drills $\rightarrow$ Executing drills $\rightarrow$ Analyzing and evaluating drills $\rightarrow$ Returning simulation results. The model frame is shown in Figure2.

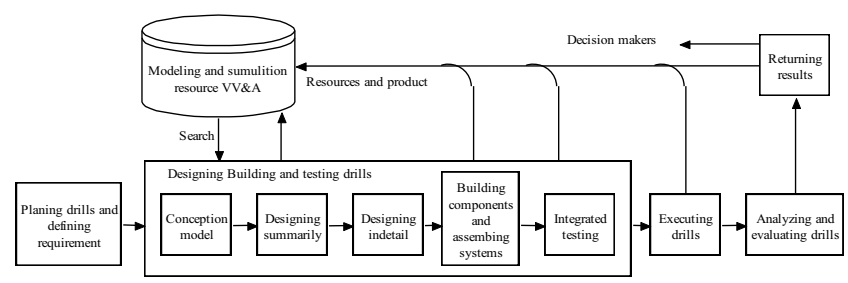

Figure2 Developing process model of DIS lifecycle VV\&A

Drill means the execution of a simulation system in its development process. The given parameters, character data, initial conditions, working personnel and external systems represent special and ordinary thoughts.

\section{B. The lifecycle $V V \& A$ developing process of simulation system based on HLA}

The total lifecycle VV\&A developing process can be concluded as six phases or steps: (1)Defining federation targets; (2)Developing federation conception models; (3)Designing federation; (4)Developing federation; (5)Integrating and testing federation; (6)Executing federation and analyzing results.

\section{The lifecycle $V V \& A$ developing process of mixed simulation system based on DIS/HLA}

DIS/HLA simulation system is a mixed and complicated simulation system. The core problem is the process of mutual information transfer and exchange. The model of developing process is shown in Figure3.

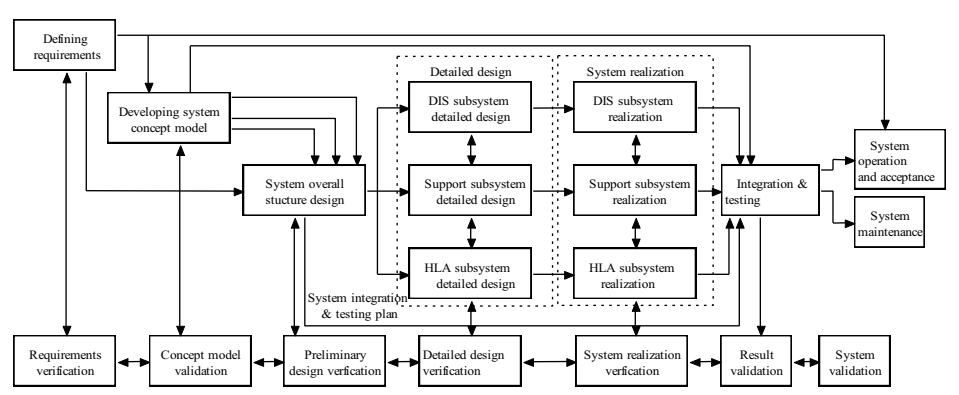

Figure3 VV\&A development process modeling of DIS/HLA simulation system in life cycle

\section{IV.THE DEVELOPING TREND OF VV\&A TECHNOLOGY}

Now, the technology and application of VV\&A has made a great progress at home and abroad. It presents some new trend as follows:

(1) With the development of information technology, the emphasis of VV\&A research has turned to the simulation system VV\&A from model verification. At present, VV\&A technology is progressing toward practicability, automation, standardization and integration to replace the partial and scattered research. There has been the "expanding VV\&A technology".

(2)The US DoD puts the effective and efficient VV\&A work in the scheme of M\&S in 2007 while gives it priority.

(3) The GM-VV which is being developed by the NATO will become the current standard of VV\&A.

(4) The western developed countries focus on the verification techniques of "black box" simulation. They launched the "M\&S international test and operation process", including the data dependency, data flow analysis and dynamic VV\&A technology (Alpha testing, operational testing, 
regression testing, statistical technology, comparing of graphics, visualization analysis and sensitivity analysis etc.). Meanwhile, they pay attention to the technology checked. In this field, the MCA has been the research hotspot.

\section{REFERENCES}

[1] Xingtang Liu, Li Liu. Simulation Science Technique and Engineering. Beijing: Sciences Publishing House, 2013.03

[2] Xingtang Liu, Bingcheng Liang. The Theory, Method \& Technique for Complex System Modeling. Beijing: Sciences Publishing House,2011.04

[3] Xingtang Liu, Li Liu. Modern Modeling and Simulation Technology. Northwestern Polytechnical University Publishing House. 2011.03

[4] Henglin Wang, Jianguo Cao. Simulation System Design and Application. Sciences Publishing House., 2003. 